\title{
Transverse-momentum distribution and nuclear modification factor for neutral pions in the forward-rapidity region in proton-lead collisions at $\sqrt{s_{N N}}=5.02 \mathrm{TeV}$
}

\author{
O. Adriani et al.* \\ (LHCf Collaboration)
}

(Received 30 March 2014; revised manuscript received 2 June 2014; published 23 June 2014)

\begin{abstract}
The transverse momentum $\left(p_{\mathrm{T}}\right)$ distribution for inclusive neutral pions in the very forward rapidity region has been measured, with the Large Hadron Collider forward detector (LHCf), in proton-lead collisions at nucleon-nucleon center-of-mass energies of $\sqrt{s_{N N}}=5.02 \mathrm{TeV}$ at the LHC. The $p_{\mathrm{T}}$ spectra obtained in the rapidity range $-11.0<y_{\text {lab }}<-8.9$ and $0<p_{\mathrm{T}}<0.6 \mathrm{GeV}$ (in the detector reference frame) show a strong suppression of the production of neutral pions after taking into account ultraperipheral collisions. This leads to a nuclear modification factor value, relative to the interpolated $p_{\mathrm{T}}$ spectra in proton-proton collisions at $\sqrt{s}=5.02 \mathrm{TeV}$, of about $0.1-0.4$. This value is compared with the predictions of several hadronic interaction Monte Carlo simulations.
\end{abstract}

DOI: 10.1103/PhysRevC.89.065209

PACS number(s): 13.85.Tp, 24.85.+p

\section{INTRODUCTION}

Measurements of particle production in hadronic interactions at high energies play an unique role in the study of strong interactions described by quantum chromodynamics (QCD). For example, as first discovered in measurements at HERA [1,2], it is still not well understood how a parton (dominantly gluon) density increases or even saturates when the momentum fraction that the parton itself carries is small (denoted as Bjorken- $x$ ). This remains true even though the understanding of the behavior of hadron constituents (partons) has been improving both theoretically and experimentally in the past few decades.

Such phenomena at small Bjorken- $x$ are known to be more visible in events at large rapidities. Since at large rapidities partons in projectile and target hadrons generally have large and small momentum fractions respectively, Bjorken- $x$ in the target should be smaller than in midrapidity events. Moreover, in case of nuclear target interactions, the parton density in the target nucleus is expected to be larger by $\sim A^{1 / 3}$. In these interactions partons in the projectile hadron would lose their energy while traveling in the dense QCD matter. Particle production mechanisms change accordingly when compared to those in nucleon-nucleon interactions.

These phenomena have been observed by several experiments at the Super Proton Synchrotron (SPS, CERN), at the Relativistic Heavy Ion Collider (RHIC, BNL), and at the Large Hadron Collider (LHC, CERN). The BRAHMS and STAR experiments at RHIC showed the modification of particle production spectra at forward rapidity $[3,4]$ by comparing the $p_{\mathrm{T}}$ spectra in deuterium-gold $(d-\mathrm{Au})$ collisions at nucleon-nucleon center-of-mass energies $\sqrt{s_{N N}}=200 \mathrm{GeV}$ with those in proton-proton $(p-p)$ collisions at center-of-mass

${ }^{*}$ Full author list given at the end of the article.

Published by the American Physical Society under the terms of the Creative Commons Attribution 3.0 License. Further distribution of this work must maintain attribution to the author(s) and the published article's title, journal citation, and DOI. energies $\sqrt{s}=200 \mathrm{GeV}$. Especially, a comparison of the experimental results between different rapidity regions by BRAHMS (pseudorapidity $\eta=2.2$ and 3.2) and STAR $(\eta=$ 4.0) indicates that particle production is strongly suppressed with increasing pseudorapidity. Also at LHC the same suppression of hadron production has been found by the ALICE and LHCb experiments, both at mid and forward rapidities $[5,6]$.

Thus one could ask, in what way does particle production take place within an extremely dense QCD matter in very forward rapidity regions? There are models that actually try to make quantitative predictions in these regions. For example under the blackbody assumption the meson production is found to be strongly suppressed as a result of limiting fragmentation with a broadened $p_{\mathrm{T}}$ distribution [7]. A suppression of particle production is also predicted using the color glass condensate formalism because of the gluon saturation dynamics [8,9]. Similarly hadronic interaction Monte Carlo (MC) simulations covering soft QCD show a modification of the $p_{\mathrm{T}}$ distributions. Experimental data that confirm the theoretical and phenomenological predictions and possibly constrain the remaining degrees of freedom in such models would thus be very welcome.

Understanding of particle production in very forward rapidity regions in nuclear target interactions is also of importance for ultrahigh-energy cosmic ray interactions, where parton density is expected to be much higher than at LHC energies due to the dependence of Bjorken- $x \propto 1 / \sqrt{s}$. In high-energy cosmic-ray observation, energy and chemical composition of primary cosmic rays are measured by analyzing the cascade showers produced by the cosmic rays interacting with the nuclei in Earth's atmosphere [10]. Secondary particles produced in the atmospheric interaction are, of course, identical to the forward emitted particles from the hadronic interactions at equivalent collision energy. In fact current modeling of particle production in nuclear interactions is limited by the available energy at the accelerators and is the cause of large systematic uncertainties in high-energy cosmic-ray physics.

The Large Hadron Collider forward (LHCf) experiment [11] is designed to measure the hadronic production cross sections of neutral particles emitted in very forward angles in $p-p$ and proton-lead ( $p-\mathrm{Pb})$ collisions at the LHC, 
including zero degree. The LHCf detectors (see Sec. II) cover a pseudorapidity range larger than 8.4 and are capable of precise measurements of the forward high-energy inclusive-particleproduction cross sections of photons, neutrons, and other neutral mesons and baryons. Therefore the LHCf experiment provides a unique opportunity to investigate the effects of high parton density which is the case in the small Bjorken- $x$ region and in $p$ - $\mathrm{Pb}$ collisions at high energies.

In the analysis presented in this paper, the focus is placed on the neutral pions ( $\pi^{0}$ 's) emitted in the direction of the proton beam (proton remnant side), the most sensitive probe of the details of the $p$ - $\mathrm{Pb}$ interactions. From the LHCf measurements, the inclusive production rate and the nuclear modification factor for $\pi^{0}$ 's in the rapidity range $-11.0<y_{\text {lab }}<-8.9$ in the detector reference frame are then derived as a function of the $\pi^{0}$ transverse momentum.

The paper is organized as follows. Section II gives a brief description of the LHCf detectors. Sections III and IV summarize the data-taking conditions and the MC simulation methodology, respectively. In Sec. V the analysis framework is described, while the factors that contribute to the systematic uncertainties are explained in Sec. VI. Finally the analysis results are presented and discussed in Sec. VII. Concluding remarks are found in Sec. VIII.

\section{THE LHCf DETECTOR}

Two independent detectors called the LHCf Arm1 and Arm 2 were assembled originally to study $p$ - $p$ collisions at the LHC $[12,13]$. In $p$-Pb collisions at $\sqrt{s_{N N}}=5.02 \mathrm{TeV}$, only the LHCf Arm2 detector was used to measure the secondary particles emitted into the proton remnant side. Hereafter the LHCf Arm2 detector is denoted as the LHCf detector for brevity. The LHCf detector has two sampling and imaging calorimeters composed of 44 radiation lengths of tungsten and 16 sampling layers of $3 \mathrm{~mm}$ thick plastic scintillators. The transverse sizes of the calorimeters are $25 \times 25 \mathrm{~mm}^{2}$ and $32 \times 32 \mathrm{~mm}^{2}$. Four X-Y layers of silicon microstrip sensors are interleaved with the layers of tungsten and scintillator in order to provide the transverse profiles of the showers. Readout pitches of the silicon microstrip sensors are $0.16 \mathrm{~mm}$ [13].

The LHCf detector was installed in the instrumentation slot of the target neutral absorber (TAN) [14] located $140 \mathrm{~m}$ in the direction of the ALICE interaction point (IP2) from the ATLAS interaction point (IP1) and at a zero-degree collision angle. The trajectories of charged particles produced at IP1 and directed towards the TAN are bent by the inner beam separation dipole magnet D1 before reaching the TAN itself. Consequently, only neutral particles produced at IP1 enter the LHCf detector. The vertical position of the LHCf detector in the TAN is manipulated so that the LHCf detector covers the pseudorapidity range from 8.4 to infinity for a beam crossing angle of $145 \mu \mathrm{rad}$; especially the smaller calorimeter covers the zero-degree collision angle. After the operations in $p$ $\mathrm{Pb}$ collisions, the LHCf detector was uninstalled from the instrumentation slot of the TAN in April, 2013.

More details on the scientific goals of the experiment are given in Ref. [11]. The construction of the LHCf detectors (Arm1 and Arm2) is reported in Refs. [12,13] and the performance of the detectors has been studied in the previous reports $[15,16]$.

\section{EXPERIMENTAL DATA-TAKING CONDITIONS}

The experimental data used for the analysis in this paper were obtained in $p$ - $\mathrm{Pb}$ collisions at $\sqrt{s_{N N}}=5.02 \mathrm{TeV}$ with a $145 \mu \mathrm{rad}$ beam crossing angle. The beam energies were $4 \mathrm{TeV}$ for protons and $1.58 \mathrm{TeV}$ per nucleon for $\mathrm{Pb}$ nuclei. Since the beam energies were asymmetric the nucleon-nucleon centerof-mass in $p$ - $\mathrm{Pb}$ collisions shifted to rapidity $=-0.465$, with the proton beam traveling at $\theta=\pi$ and the $\mathrm{Pb}$ beam at $\theta=0$.

Data used in this analysis were taken in three different runs. The first (LHC Fill 3478) was taken on January 21, 2013 from 02:14 to 03:53. The second and third runs (LHC Fill 3481) were taken on January 21, 2013 from 21:03 to 23:36 and January 22, 2013 from 03:47 to 04:48, respectively. The integrated luminosity of the data was $0.63 \mathrm{nb}^{-1}$ after correcting for the live time of data acquisition systems [17]. The average live time percentages for LHC Fills 3478 and 3481 were $12.1 \%$ and $6.3 \%$ respectively, the smaller live time percentage in Fill 3481 relative to Fill 3478 being due to a difference in the instantaneous luminosities. These three runs were taken with the same data acquisition configuration. In all runs the trigger scheme was essentially identical to that used in $p$ - $p$ collisions at $\sqrt{s}=7 \mathrm{TeV}$. The trigger efficiency was greater than $99 \%$ for photons with energies $E>100 \mathrm{GeV}$ [16].

The multihit events that have more than one shower event in a single calorimeter may appear due to pileup interactions in the same bunch crossing, and then could potentially cause a bias in the $p_{\mathrm{T}}$ spectra. However, considering the acceptance of the LHCf detector for inelastic collisions $\sim 0.035$, the multihit probability due to the effects of pileup is estimated to be only $0.4 \%$ and is therefore producing a negligible effect. Detailed discussions of pileup effects and background events from collisions between the beam and residual gas molecules in the beam tube can be found in previous reports [16,18].

Also beam divergence can cause a smeared beam spot at the TAN, leading to a bias in the measured $p_{\mathrm{T}}$ spectra. The beam divergence at IP1 was $\varepsilon / \beta^{*}=32 \mu \mathrm{rad}$ [19] for the three fills mentioned, corresponding to a beam spot size at the TAN of roughly $\sigma_{\mathrm{TAN}}=4.5 \mathrm{~mm}$. The effect of a nonzero beam spot size at the TAN is evaluated by comparing two $p_{\mathrm{T}}$ spectra predicted by toy MC simulations: one assuming a beam spot size of zero and another assuming that the beam axis positions fluctuates following a Gaussian distribution with $\sigma_{\mathrm{TAN}}=4.5 \mathrm{~mm}$. The $\pi^{0}$ yield at $p_{\mathrm{T}}=0.6 \mathrm{GeV}$ is found to increase by a factor of 1.8 at most. This effect is taken into account in the final results to the $p_{\mathrm{T}}$ spectra.

\section{MONTE CARLO SIMULATIONS METHODOLOGY}

MC simulations were performed in two steps: (I) $p-\mathrm{Pb}$ interaction event generation at IP1 explained in Sec. IV A and (II) particle transport from IP1 to the LHCf detector and consequent simulation of the response of the LHCf detector (Sec. IV B).

MC simulations were then used for the validation of reconstruction algorithms and cut criteria, and the estimation of systematic uncertainties follow steps (I) and (II). These MC 
simulations are denoted as reference MC simulations. On the other hand, MC simulations used only for comparisons with measurement results in Sec. VII are limited to step (I) only, since the final $p_{\mathrm{T}}$ spectra in Sec. VII are already corrected for detector responses and eventual reconstruction bias.

\section{A. Signal modeling}

Whenever the impact parameter of proton and $\mathrm{Pb}$ is smaller than the radius of each particle, soft-QCD induced events are produced. These $p-\mathrm{Pb}$ interactions at $\sqrt{s_{N N}}=5.02 \mathrm{TeV}$ and the resulting flux of secondary particles emitted into the forward rapidity region with their kinematics are simulated using various hadronic interaction models (DPMJET 3.04 [20], QGSJET II-03 [21], and EPOS 1.99 [22]). DPMJET 3.04 also takes into account the Fermi motion of the nucleons in the $\mathrm{Pb}$ nucleus and the Cronin effect [23]. Fermi motion enhances the $\pi^{0}$ yields at most by $5 \%$ in the LHCf $p_{\mathrm{T}}$ covered range $p_{\mathrm{T}}<0.6 \mathrm{GeV}$, while the Cronin effect is not significant in this $p_{\mathrm{T}}$ range.

On the other hand, when the impact parameter is larger than the overlapping radii of each particle, so-called ultraperipheral collisions (UPCs) can occur. In UPCs virtual photons are emitted by the relativistic $\mathrm{Pb}$ nucleus which can then collide with the proton beam [24]. The energy spectrum of these virtual photons follows the Weizsäcker-Williams approximation [25]. The SOPHIA [26] MC generator is used to simulate the photon-proton interaction in the rest frame of the proton and then the secondary particles generated by SOPHIA are boosted along the proton beam. For heavy nuclei with radius $R_{\mathrm{A}}$, the virtuality of the photon $\left|q^{2}\right|<\left(\hbar c / R_{\mathrm{A}}\right)^{2}$ can be neglected and the photons are regarded as real photons in the simulation in this analysis.

In these MC simulations, $\pi^{0}$ 's from short-lived particles that decay within $1 \mathrm{~m}$ from IP1, mostly $\eta$ mesons decaying into $3 \pi^{0}$ ( $\lesssim 10 \%$ relative to all $\pi^{0}$ 's), are also accounted for consistently with the treatment of the experimental data.

\section{B. Simulation of particle transport from IP1 to the LHCf detector and of the detector response}

The generated secondary particles are transported in the beam pipe from IP1 to the TAN, taking into account the bending of charged particles' trajectory by the Q1 quadrupole and the D1 beam separation dipole, particle decays, and particle interactions with the beam pipe wall and the Y-shaped beam-vacuum-transition chamber made of copper.

Finally the showers produced in the LHCf detector by the particles arriving at the TAN and the detector response are simulated with the COSMOS and EPICS libraries [27]. The detector position survey data and random fluctuations due to electrical noise are taken into account. The simulations of the LHCf detector are tuned to the test beam data taken at SPS, CERN in 2007 and 2012 [15,28].

\section{ANALYSIS FRAMEWORK}

\section{A. $\pi^{\mathbf{0}}$ event reconstruction and selection}

Since $\pi^{0}$ 's decay into two photons very close to their point of creation at IP1, each photon's direction is geometrically calculated using the impact coordinates at the LHCf detector
TABLE I. Summary of the criteria for the selection of the $\pi^{0}$ sample.

\begin{tabular}{ll}
\hline Incident position & Within $2 \mathrm{~mm}$ from the edge of calorimeter \\
Energy threshold & $E_{\text {photon }}>100 \mathrm{GeV}$ \\
Number of hits & Single-hit in each calorimeter \\
PID & Photon-like in each calorimeter \\
\hline
\end{tabular}

and the distance between IP1 and the detector itself. Photon four-momentum is then derived by combining the photon's energy as measured by the calorimeter with the previously obtained angle of emission. Candidate $\pi^{0}$ 's are selected from events where the invariant mass of the two photons detected is within a narrow window around the $\pi^{0}$ rest mass.

The $\pi^{0}$ events are then classified in two categories: singlehit $\pi^{0}$ and multihit $\pi^{0}$ events. The former is defined as having a single photon in each of the two calorimeter towers only, while a multihit $\pi^{0}$ event is defined as a single $\pi^{0}$ accompanied by at least one additional background particle (usually a photon or a neutron) in one of the two calorimeters. In the analysis presented here, events having two particles within the same calorimeter tower (multihit events) are rejected when the energy deposit of the background particle is above a certain threshold [29]. Mostly then, only single-hit $\pi^{0}$ events are considered in this analysis. The final inclusive production rates reported at the end are corrected for this cut as described in Sec. V C.

Given the geometrical acceptance of the LHCf detector and to ensure a good event reconstruction efficiency, the rapidity and $p_{\mathrm{T}}$ range of the $\pi^{0}$ 's are limited to $-11.0<y_{\text {lab }}<$ -8.9 and $p_{\mathrm{T}}<0.6 \mathrm{GeV}$, respectively. The reconstructed invariant mass of the reference MC simulations peaks at $134.8 \pm 0.2 \mathrm{MeV}$ and reproduces well the measured data which peaks at $134.7 \pm 0.1 \mathrm{MeV}$, reproducing the $\pi^{0}$ mass. The uncertainties given for the mass peaks are statistical only.

Standard reconstruction algorithms used for this analysis are described in Refs. [16,29] and the $\pi^{0}$ event selection criteria that are applied prior to the reconstruction of the $\pi^{0}$ kinematics are summarized in Table I. Systematic uncertainties are discussed in Sec. VI.

\section{B. Background subtraction}

Background contamination of the $\pi^{0}$ events from hadronic events and the causal coincidence of two photons not originating from the decay of a single $\pi^{0}$ are taken into account by subtracting the relevant contribution using a sideband method [29].

Figure 1 shows the reconstructed two-photon invariant mass distribution of the experimental data in the rapidity range $-9.4<y_{\text {lab }}<-9.2$. The sharp peak around $135 \mathrm{MeV}$ is due to $\pi^{0}$ events. The solid curve indicates the best fit of a composite physics model to the invariant mass distribution of the data: an asymmetric Gaussian distribution for the signal component and a third-order Chebyshev polynomial function for the background component (dashed curve). The signal window is defined as the invariant mass region within the two solid arrows shown in Fig. 1, where the lower and upper limits 


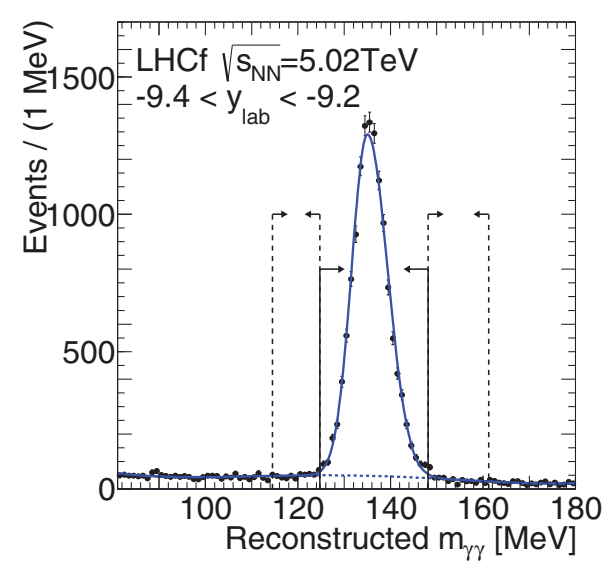

FIG. 1. (Color online) Reconstructed invariant mass distribution within the rapidity range from -9.4 to -9.2 . The solid curve shows the best-fit composite physics model to the invariant mass distribution. The dashed curve indicates the background component. Solid and dashed lines indicate the signal and background windows, respectively.

are given by $\hat{m}-3 \sigma_{l}$ and $\hat{m}+3 \sigma_{u}$, respectively. $\hat{m}$ denotes the expected mean, and $\sigma_{l}$ and $\sigma_{u}$ denote $1 \sigma$ deviations for lower and upper sides of the signal component, respectively. The signal-rich rapidity- $p_{\mathrm{T}}$ distributions are obtained by the events contained inside of the signal window. The remaining contribution of background events in the signal window is eliminated using the rapidity- $p_{\mathrm{T}}$ distributions obtained from the background window, constructed from the two sideband regions, $\left[\hat{m}-6 \sigma_{l}, \hat{m}-3 \sigma_{l}\right]$ and $\left[\hat{m}+3 \sigma_{u}, \hat{m}+6 \sigma_{u}\right]$, that are defined as the invariant mass regions within the dashed arrows in Fig. 1.

\section{Corrections to the $p_{\mathrm{T}}$ spectra}

The raw rapidity- $p_{\mathrm{T}}$ distributions must be corrected for (1) the reconstruction inefficiency and the smearing caused by finite position and energy resolutions, (2) geometrical acceptance and branching ratio of $\pi^{0}$ decay, and (3) the loss due to multihit $\pi^{0}$.

First, an iterative Bayesian method [30] is used to simultaneously correct for both the reconstruction inefficiency and the smearing. The unfolding procedure for the data is found in paper [29].

Next, a correction for the limited aperture of the LHCf calorimeters must be applied. The correction is derived from the rapidity- $p_{\mathrm{T}}$ phase space. The determination of the correction coefficients follows the same method used in the $\pi^{0}$ event analysis in $p$ - $p$ collisions at $\sqrt{s}=7 \mathrm{TeV}$ [29].

Finally, the loss of multihit $\pi^{0}$ events, briefly mentioned in Sec. V A, is corrected for using the MC event generators. A range of ratios of multihit plus single-hit to single-hit $\pi^{0}$ events is estimated using three different hadronic interaction models (DPMJET 3.04, QGSJET II-03, and EPOS 1.99) for each rapidity and $p_{\mathrm{T}}$ range. The observed $p_{\mathrm{T}}$ spectra are then multiplied by the average of these ratios and the contribution to the systematic uncertainty is derived from the observed variations among the interaction models. The estimated range of the flux of multihit $\pi^{0}$ events lies within a range $0 \%-10 \%$ of the flux of single-hit
TABLE II. Summary of the systematic uncertainties. Numerical values indicate the maximum variation of bin contents in the $p_{\mathrm{T}}$ spectra.

Energy scale

$5 \%-20 \%$

Particle identification

$0 \%-20 \%$

Offset of beam axis

$5 \%-20 \%$

Single-hit selection

$3 \%$

Position-dependent correction

$5 \%-30 \%$

Luminosity

$20 \%$

$\pi^{0}$ events. The single-hit $\pi^{0}$ spectra are then corrected to represent the inclusive $\pi^{0}$ production spectra.

All the procedures were verified using the reference MC simulations mentioned in Sec. IV.

\section{SYSTEMATIC UNCERTAINTIES}

We follow the same approach to estimate the systematic uncertainties as in Ref. [29]. Since systematic uncertainties on particle identification, single-hit selection, and positiondependent corrections for both shower leakage and light yield of the calorimeter are independent of the beam energy and type, the systematic errors are taken directly from Ref. [29].

Other terms deriving from the energy scale, beam axis offset, and luminosity are updated consistently to the current understanding of the LHCf detector and the beam configuration in $p-\mathrm{Pb}$ collisions. These terms are discussed in the following subsections. Table II summarises the systematic uncertainties for the analysis.

\section{A. Energy scale}

The uncertainty on the measured photon energy was investigated in the beam test at SPS [15] and also by performing a calibration with a radioactive source [12]. The estimated uncertainty on the photon energy from these tests is valued at $3.5 \%$. This uncertainty is dominated by the conversion factors that relate measured charge to deposited energy [15], and in fact the reconstructed invariant mass of two photons reproduces the $\pi^{0}$ rest mass within an uncertainty of $3.5 \%$ as shown in Fig. 1.

The systematic shift of bin contents due to energy scale uncertainties is estimated using two different $p_{\mathrm{T}}$ spectra in which the photon energy is artificially scaled to the two extremes $( \pm 3.5 \%)$. The ratios of the two extreme spectra to the nonscaled spectrum are assigned as systematic shifts of bin contents for each bin.

\section{B. Beam axis offset}

The projected position of the proton beam axis on the LHCf detector (beam center) varies from Fill to Fill owing to the beam configuration, beam transverse position, and crossing angles, at IP1. The beam center on the LHCf detector can be determined by two methods: first we use the distribution of incident particle positions as measured by the LHCf detector, and second we also use the information from the beam position monitors (BPMSW) installed $\pm 21 \mathrm{~m}$ from IP1 [31]. 
From analysis results for $p$ - $p$ collisions at $\sqrt{s}=7 \mathrm{TeV}$, the beam center positions obtained by the two methods applied to LHC Fills 1089-1134 were found to be consistent within 1 $\mathrm{mm}$. The systematic shifts to the $p_{\mathrm{T}}$ spectra are then evaluated by taking the ratio of spectra with the beam center displaced by $\pm 1 \mathrm{~mm}$ to spectra with no displacement present. The fluctuations of the beam center position modify the $p_{\mathrm{T}}$ spectra by $5 \%-20 \%$ depending on the rapidity range.

\section{Luminosity}

The luminosity value used for the analysis is derived from on the online information provided by the ATLAS experiment. Since there is currently no robust estimation of the luminosity error by the ATLAS experiment, we assign a conservative $\pm 20 \%$ to the uncertainty. A more precise estimation of the luminosity will be reported in future by the ATLAS Collaboration.

\section{RESULTS AND DISCUSSION}

\section{A. The QCD induced transverse momentum distribution}

The $p_{\mathrm{T}}$ spectra obtained from the data analysed are presented in Fig. 2. The spectra are categorized into six ranges of rapidity $y_{\mathrm{lab}}:[-9.0,-8.9],[-9.2,-9.0],[-9.4,-9.2]$, $[-9.6,-9.4],[-10.0,-9.6]$, and $[-11.0,-10.0]$. The spectra have all the corrections discussed in Sec. VC applied. The inclusive $\pi^{0}$ production rate is given as

$$
\frac{1}{\sigma_{\text {inel }}^{\mathrm{pPb}}} E \frac{d^{3} \sigma^{\mathrm{pPb}}}{d p^{3}}=\frac{1}{N_{\text {inel }}^{\mathrm{pPb}}} \frac{d^{2} N^{\mathrm{pPb}}\left(p_{\mathrm{T}}, y\right)}{2 \pi p_{\mathrm{T}} d p_{\mathrm{T}} d y} .
$$

where $\sigma_{\text {inel }}^{\mathrm{pPb}}$ is the inelastic cross section for $p$-Pb collisions at $\sqrt{s_{N N}}=5.02 \mathrm{TeV}$ and $E d^{3} \sigma^{\mathrm{pPb}} / d p^{3}$ is the inclusive cross section of $\pi^{0}$ production. The number of inelastic $p-\mathrm{Pb}$ collisions, $N_{\text {inel }}^{\mathrm{pPb}}$, used for normalizing the production rates of Fig. 2 is calculated from $N_{\text {inel }}^{\mathrm{pPb}}=\sigma_{\text {inel }}^{\mathrm{pPb}} \int \mathcal{L} d t$, assuming an inelastic $p-\mathrm{Pb}$ cross section $\sigma_{\text {inel }}^{\mathrm{pPb}}=2.11 \mathrm{~b}$. The value for $\sigma_{\text {inel }}^{\mathrm{pPb}}$ is derived from the inelastic $p-p$ cross section $\sigma_{\text {inel }}^{\mathrm{pp}}$ and the Glauber multiple collision model $[32,33]$. Using the integrated luminosities shown in Sec. III, $N_{\text {inel }}^{\mathrm{pPb}}$ is $9.33 \times 10^{7}$. $d^{2} N^{\mathrm{pPb}}\left(p_{\mathrm{T}}, y\right)$ is the number of $\pi^{0}$ 's detected in the transverse momentum interval $\left(d p_{\mathrm{T}}\right)$ and the rapidity interval $(d y)$ with all corrections applied.

In Fig. 2, the filled circles represent the data from the LHCf experiment. The error bars and shaded rectangles indicate the one-standard-deviation statistical and total systematic uncertainties respectively. The total systematic uncertainties are given by adding all uncertainty terms except the one for luminosity in quadrature. The vertical dashed lines shown for the rapidity ranges greater than -9.2 indicate the $p_{\mathrm{T}}$ threshold of the LHCf detector due to the photon energy threshold and the geometrical acceptance of the detector. The contribution from UPCs is presented as open squares (normalized to $1 / 2$
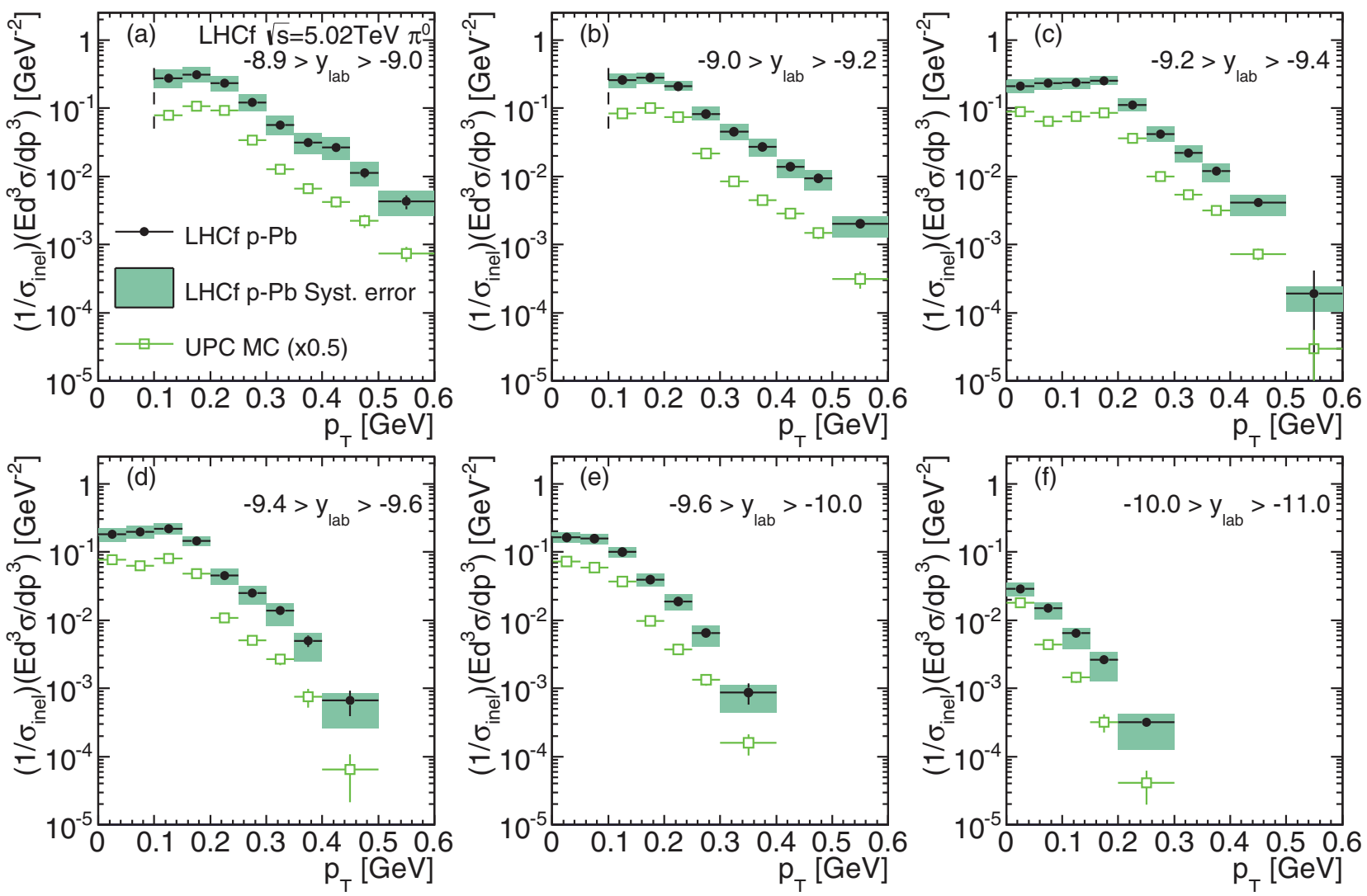

FIG. 2. (Color online) Experimental $p_{\mathrm{T}}$ spectra of the LHCf detector (filled circles). Error bars and shaded rectangles indicate the statistical and systematic uncertainties respectively. The open squares indicate the estimated contribution from UPCs. 

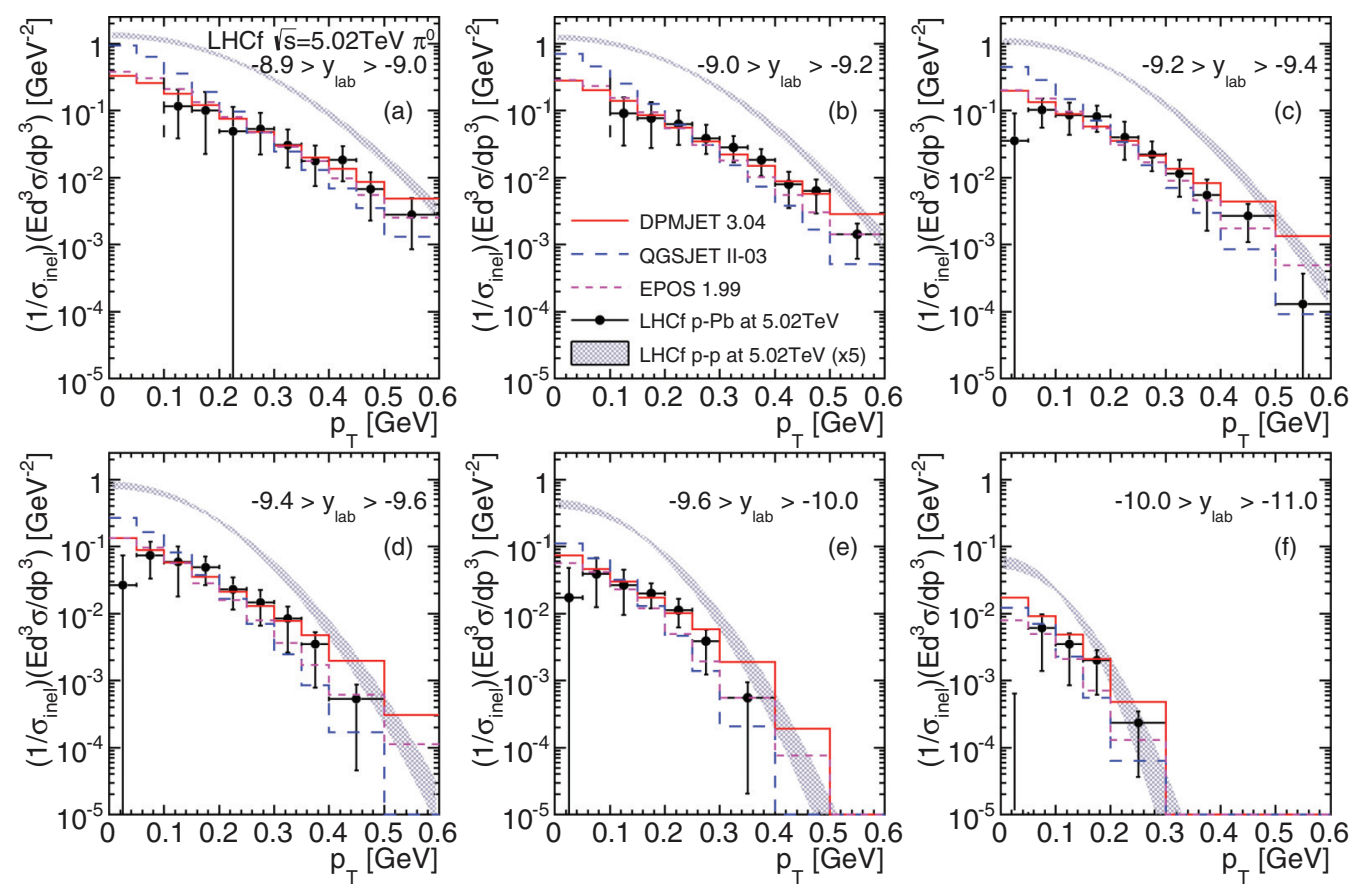

FIG. 3. (Color online) Experimental $p_{\mathrm{T}}$ spectra measured by LHCf after the subtraction of the UPC component (filled circles). Error bars indicate the total uncertainties incorporating both statistical and systematic uncertainties. Hadronic interaction models predictions and derived spectra for $p$ - $p$ collisions at 5.02 TeV are also shown (see text for details).

for visibility). This UPC contribution is estimated with the MC simulations introduced in Sec. IV A using the UPC cross section from [34].

To obtain the soft-QCD component, the UPC contribution must be subtracted from the measured $p_{\mathrm{T}}$ spectra. This is achieved by simply subtracting, point by point, the UPC induced $p_{\mathrm{T}}$ spectra (open squares in Fig. 2) from the total $p_{\mathrm{T}}$ spectra (filled circles in Fig. 2). Uncertainties in the subtracted results are obtained by adding the statistical and systematic errors in quadrature. The theoretical uncertainty on the UPC estimation $\pm 5 \%$ derives mainly from the knowledge of the virtual photon flux given by the relativistic $\mathrm{Pb}$ nucleus and of the virtual photon-proton cross section [34]. The inclusive production rates of $\pi^{0}$ s measured by LHCf after the subtraction of the UPC component are summarized in Appendix A.

Figure 3 shows the LHCf data $p_{\mathrm{T}}$ spectra after subtraction of the UPC component (filled circles). The sizes of the error bars correspond to $68 \%$ confidence intervals (including both statistical and systematic uncertainties). The $p_{\mathrm{T}}$ spectra in $p-\mathrm{Pb}$ collisions at $5.02 \mathrm{TeV}$ predicted by the hadronic interaction models, DPMJET 3.04 (solid line, red), QGSJET II-03 (dashed line, blue), and EPOS 1.99 (dotted line, magenta), are also shown in the same figure for comparison. Predictions by the three hadronic interaction models do not include the UPC component. The experimental $p_{\mathrm{T}}$ spectra are corrected for detector response, event selection, and geometrical acceptance efficiencies, so that the $p_{\mathrm{T}}$ spectra of the interaction models can be compared directly to the experimental spectra.

In Fig. 3, among the predictions given by the hadronic interaction models tested here, DPMJET 3.04 and EPOS 1.99 show a good overall agreement with the LHCf measurements.
However QGSJET II-03 predicts softer $p_{\mathrm{T}}$ spectra than the LHCf measurements and the other two hadronic interaction models. Similar features of these hadronic interaction models are also seen in $p$ - $p$ collisions at $\sqrt{s}=7 \mathrm{TeV}$ [29].

In Fig. 3 , the $p_{\mathrm{T}}$ spectra in $p$ - $p$ collisions at $\sqrt{s}=5.02 \mathrm{TeV}$ are also added and will be useful for the derivation of the nuclear modification factor described later in Sec. VII C. These spectra are multiplied by a factor of 5 for visibility. The derivation of the $p_{\mathrm{T}}$ spectra in $p$ - $p$ collisions at $\sqrt{s}=5.02 \mathrm{TeV}$ is explained in detail in Appendix B.

\section{B. Average transverse momentum}

The average transverse momentum, $\left\langle p_{\mathrm{T}}\right\rangle$, can be obtained by fitting an empirical function to the $p_{\mathrm{T}}$ spectra in Fig. 3 for each rapidity range. Two distributions to parametrize the $p_{\mathrm{T}}$ spectra were chosen among the several proposed in literature: an exponential and a Gaussian. Detailed descriptions of the parametrization and derivation of $\left\langle p_{\mathrm{T}}\right\rangle$ can be found in Ref. [29].

For example, the upper panel in Fig. 4 shows the experimental $p_{\mathrm{T}}$ spectra (filled circles) and the best fit with the exponential (dashed curve, blue) and with the Gaussian distribution (dotted curve, red) in the rapidity range $-9.2>$ $y_{\text {lab }}>-9.4$. The bottom panel in Fig. 4 shows best-fit ratio to the experimental data, for exponential (blue open triangles) and Gaussian distributions (red open circles). Error bars indicate the statistical and systematic uncertainties in the both panels.

On the other hand, $\left\langle p_{\mathrm{T}}\right\rangle$ can be simply estimated by numerically integrating the $p_{\mathrm{T}}$ spectra in Fig. 3. In this approach, $\left\langle p_{\mathrm{T}}\right\rangle$ is obtained over the rapidity range $-9.2>$ $y_{\text {lab }}>-10.0$ where the $p_{\mathrm{T}}$ spectra are available down to 


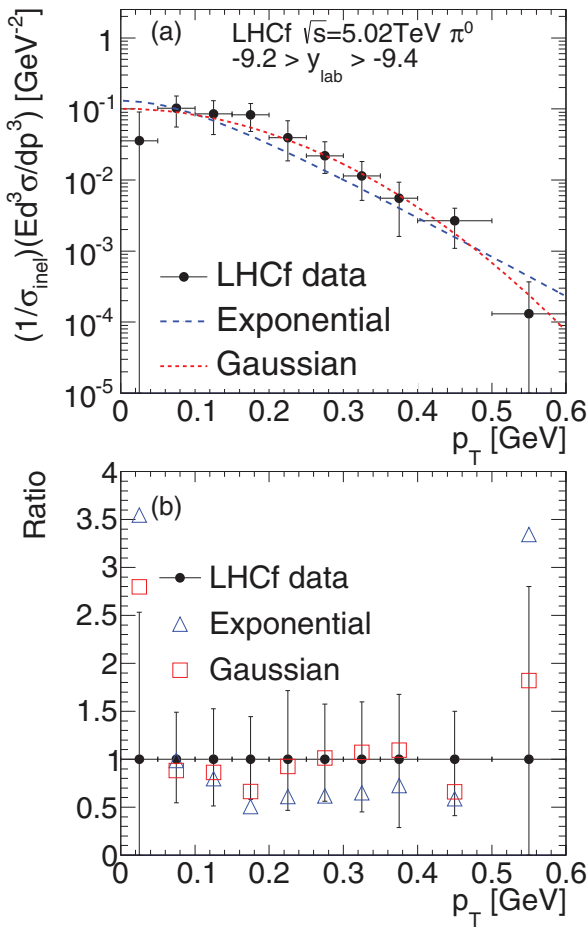

FIG. 4. (Color online) (a) Experimental $p_{\mathrm{T}}$ spectra (filled circles and error bars), and the best-fit exponential (dashed curve) and Gaussian distributions (dotted curve). (b) Ratios of the best-fit exponential and Gaussian distribution to the experimental data (open triangles or open boxes) with statistical and systematic uncertainties (filled circles and error bars.)

$0 \mathrm{GeV}$. The data in the rapidity range $-10.0>y_{\text {lab }}>-11.0$ are not used here, since the bin content in $0<p_{\mathrm{T}}<0.05 \mathrm{GeV}$ is negative due to the subtraction of UPCs. Although the interval for the numerical integration is bounded from above by $p_{\mathrm{T}}^{\text {upper }}$, the high- $p_{\mathrm{T}}$ tail contribution to $\left\langle p_{\mathrm{T}}\right\rangle$ is negligibly small.

The values of $\left\langle p_{\mathrm{T}}\right\rangle$ obtained by the three methods are in good agreement within the uncertainties. The specific values of $\left\langle p_{\mathrm{T}}\right\rangle$ for this paper, $\left\langle p_{\mathrm{T}}\right\rangle_{\mathrm{LHCf}}$, are defined as follows. For the rapidity range $-9.2>y_{\text {lab }}>-10.0$, the values of $\left\langle p_{\mathrm{T}}\right\rangle_{\mathrm{LHCf}}$ are taken from the weighted average of $\left\langle p_{\mathrm{T}}\right\rangle$ from the exponential fit, the Gaussian fit, and the numerical integration. The uncertainty related to a possible bias of the $\left\langle p_{\mathrm{T}}\right\rangle$ extraction methods is derived from the largest value among the three methods. For the other rapidity ranges to where the numerical integration is not applicable, the weighted mean and the uncertainty are obtained following the same method above but using only the exponential and the Gaussian fits. Best-fit results for the three approaches mentioned above and the values of $\left\langle p_{\mathrm{T}}\right\rangle_{\mathrm{LHCf}}$ are summarized in Table III.

Figure 5 shows the $\left\langle p_{\mathrm{T}}\right\rangle_{\mathrm{LHCf}}$ and the predictions by hadronic interaction models as a function of the rapidity $y_{\text {lab. The }}$ average $p_{\mathrm{T}}$ of the hadronic interaction models is calculated by numerical integration. DPMJET 3.04 reproduces quite well the LHCf measurements $\left\langle p_{\mathrm{T}}\right\rangle_{\mathrm{LHCf}}$, while EPOS 1.99 is slightly softer than both DPMJET 3.04 and the LHCf measurements. QGSJET II-03 shows the smallest $\left\langle p_{\mathrm{T}}\right\rangle$ among the three models and the LHCf measurements. These tendencies are also found in Fig. 3.

\section{Nuclear modification factor}

Finally the nuclear modification factor $R_{\mathrm{pPb}}$ is derived. This factor quantifies the $p_{\mathrm{T}}$ spectra modification caused by nuclear effects in $p-\mathrm{Pb}$ collisions. The nuclear modification factor is defined as

$$
R_{\mathrm{pPb}} \equiv \frac{\sigma_{\text {inel }}^{\mathrm{pp}}}{\left\langle N_{\text {coll }}\right\rangle \sigma_{\text {inel }}^{\mathrm{pPb}}} \frac{E d^{3} \sigma^{\mathrm{pPb}} / d p^{3}}{E d^{3} \sigma^{\mathrm{pp}} / d p^{3}},
$$

where $E d^{3} \sigma^{\mathrm{pPb}} / d p^{3}$ and $E d^{3} \sigma^{\mathrm{pp}} / d p^{3}$ are the inclusive cross sections of $\pi^{0}$ production in $p-\mathrm{Pb}$ and $p$ - $p$ collisions at $5.02 \mathrm{TeV}$ respectively. The UPC component is already subtracted in $\sigma^{\mathrm{pPb}}$. The uncertainty on $\sigma_{\text {inel }}^{\mathrm{pPb}}$ is estimated to be $\pm 5 \%$ by comparing the $\sigma_{\text {inel }}^{\mathrm{pPb}}$ value with other calculations and experimental results presented in Refs. [35,36]. The average number of binary nucleon-nucleon collisions in a $p$ - $\mathrm{Pb}$ collision, $\left\langle N_{\text {coll }}\right\rangle=6.9$, is obtained from MC simulations using the Glauber model [33]. The uncertainty in $\sigma_{\text {inel }}^{\mathrm{pp}} /\left\langle N_{\text {coll }}\right\rangle$ is estimated by varying the parameters in the calculation with the Glauber model $[5,37]$ (where the cancellation of the uncertainties in $\sigma_{\text {inel }}^{\mathrm{pp}}$ and $\left\langle N_{\text {coll }}\right\rangle$ is taken into account) and is of the order of $\pm 3.5 \%$. Finally the quadratic sum of the uncertainties in $\sigma_{\text {inel }}^{\mathrm{pPb}}$ and $\sigma_{\text {inel }}^{\mathrm{pp}} /\left\langle N_{\text {coll }}\right\rangle$ is added to $R_{\mathrm{pPb}}$.

Since there are no data at $\sqrt{s}=5.02 \mathrm{TeV}$ for $p$ - $p$ collisions, $E d^{3} \sigma^{\mathrm{pp}} / d p^{3}$ is derived by scaling the $p_{\mathrm{T}}$ spectra taken in $p$ - $p$ collisions at 7 and $2.76 \mathrm{TeV}$. The derivation follows three steps. First (I) the $\left\langle p_{\mathrm{T}}\right\rangle$ values at $5.02 \mathrm{TeV}$ are estimated by interpolating the $\left\langle p_{\mathrm{T}}\right\rangle$ values at 7 and $2.76 \mathrm{TeV}$, assuming that

TABLE III. The average $\pi^{0}$ transverse momenta for the rapidity range $-8.9>y_{\text {lab }}>-11.0$ estimated by the three approaches (exponential, Gaussian, and numerical integration). Combined results using the three approaches are denoted as LHCf results.

\begin{tabular}{|c|c|c|c|c|c|c|c|c|c|c|c|}
\hline \multirow[t]{2}{*}{ Rapidity } & \multicolumn{3}{|c|}{ Exponential fit } & \multicolumn{3}{|c|}{ Gaussian fit } & \multicolumn{3}{|c|}{ Numerical integration } & \multicolumn{2}{|c|}{ LHCf analysis } \\
\hline & $\chi^{2}$ (dof) & $\begin{array}{c}\left\langle p_{\mathrm{T}}\right\rangle \\
(\mathrm{MeV})\end{array}$ & $\begin{array}{c}\text { Stat. error } \\
(\mathrm{MeV})\end{array}$ & $\chi^{2}(\mathrm{dof})$ & $\begin{array}{c}\left\langle p_{\mathrm{T}}\right\rangle \\
(\mathrm{MeV})\end{array}$ & $\begin{array}{c}\text { Stat. error } \\
(\mathrm{MeV})\end{array}$ & $\begin{array}{l}p_{\mathrm{T}}^{\text {upper }} \\
(\mathrm{GeV})\end{array}$ & $\begin{array}{c}\left\langle p_{\mathrm{T}}\right\rangle \\
(\mathrm{MeV})\end{array}$ & $\begin{array}{l}\text { Stat. error } \\
(\mathrm{MeV})\end{array}$ & $\begin{array}{c}\left\langle p_{\mathrm{T}}\right\rangle_{\mathrm{LHCf}} \\
(\mathrm{MeV})\end{array}$ & $\begin{array}{c}\text { Syst. error } \\
(\mathrm{MeV})\end{array}$ \\
\hline$[-8.9,-9.0]$ & $0.9(7)$ & 249.1 & 36.8 & $0.8(7)$ & 258.5 & 27.9 & & & & 255.3 & 36.8 \\
\hline$[-9.0,-9.2]$ & $2.0(7)$ & 221.1 & 20.4 & $0.5(7)$ & 239.5 & 16.6 & & & & 232.3 & 20.4 \\
\hline$[-9.2,-9.4]$ & $7.6(8)$ & 188.7 & 13.4 & $2.7(8)$ & 196.4 & 8.6 & 0.6 & 193.3 & 13.2 & 194.4 & 13.4 \\
\hline$[-9.4,-9.6]$ & $4.8(6)$ & 181.8 & 16.3 & $1.6(6)$ & 187.0 & 12.7 & 0.5 & 184.6 & 14.9 & 185.7 & 16.3 \\
\hline$[-9.6,-10.0]$ & $3.7(5)$ & 153.0 & 16.3 & $1.5(5)$ & 153.7 & 12.3 & 0.4 & 152.2 & 13.9 & 153.9 & 16.3 \\
\hline$[-10.0,-11.0]$ & $<0.1(2)$ & 115.1 & 22.2 & $<0.1(2)$ & 117.5 & 17.5 & & & & 116.6 & 22.2 \\
\hline
\end{tabular}




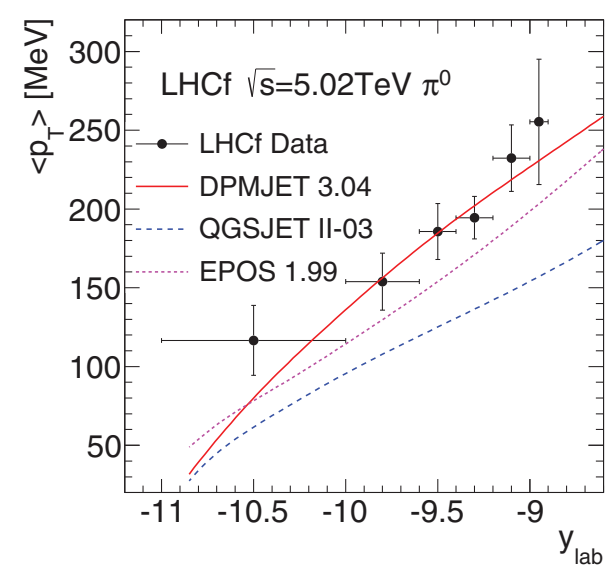

FIG. 5. (Color online) Average $p_{\mathrm{T}}$ as a function of rapidity $y_{\text {lab }}$. Filled circles indicate the LHCf data. The predictions of hadronic interaction models are shown (solid curve DPMJET 3.04, dashed curve QGSJET II-03, and dotted curve EPOS 1.99).

the Feynman scaling of $\left\langle p_{\mathrm{T}}\right\rangle$ is only a function of rapidity. Then (II) the absolute normalizations of the $p_{\mathrm{T}}$ spectra at $5.02 \mathrm{TeV}$ are determined by applying the measured absolute normalizations at $7 \mathrm{TeV}$ directly to those at $5.02 \mathrm{TeV}$. Finally (III) the $p_{\mathrm{T}}$ spectra at $5.02 \mathrm{TeV}$ are derived assuming that the $p_{\mathrm{T}}$ spectra follow a Gaussian distribution with width $2\left\langle p_{\mathrm{T}}\right\rangle / \sqrt{\pi}$ [obtained in step (I)] and using the normalizations obtained in step (II). The rapidity shift -0.465 explained in Sec. III is also taken into account in the $p_{\mathrm{T}}$ spectrum at $5.02 \mathrm{TeV}$. The details of the procedure are discussed in Appendix B.

Figure 6 shows the nuclear modification factors $R_{\mathrm{pPb}}$ from the LHCf measurements and the predictions by hadronic interaction models DPMJET 3.04 (red solid line), QGSJET II-03 (blue dashed line), and EPOS 1.99 (magenta dotted line). The LHCf measurements, although having a large uncertainty which increases with $p_{\mathrm{T}}$ (mainly due to systematic uncertainties in $p-\mathrm{Pb}$ collisions at $5.02 \mathrm{TeV}$ ), show a strong suppression with $R_{\mathrm{pPb}}$ equal to 0.1 at $p_{\mathrm{T}} \approx 0.1 \mathrm{GeV}$ rising to 0.3 at $p_{\mathrm{T}} \approx 0.6 \mathrm{GeV}$. All hadronic interaction models predict small values of $R_{\mathrm{pPb}} \approx 0.1$, and they show an overall good agreement with the LHCf measurements within the uncertainty. Clearly other analyses which are more sensitive to exclusive $\pi^{0}$ signals are needed, for example diffractive dissociation, to investigate the reason for this strong suppression. However, the measured $R_{\mathrm{pPb}}$ dependency on $p_{\mathrm{T}}$ and rapidity may hint to an understanding of the breakdown of the $\pi^{0}$ production mechanism.

\section{CONCLUSIONS}

The inclusive production of neutral pions in the rapidity range $-8.9>y_{\text {lab }}>-11.0$ has been measured by the LHCf
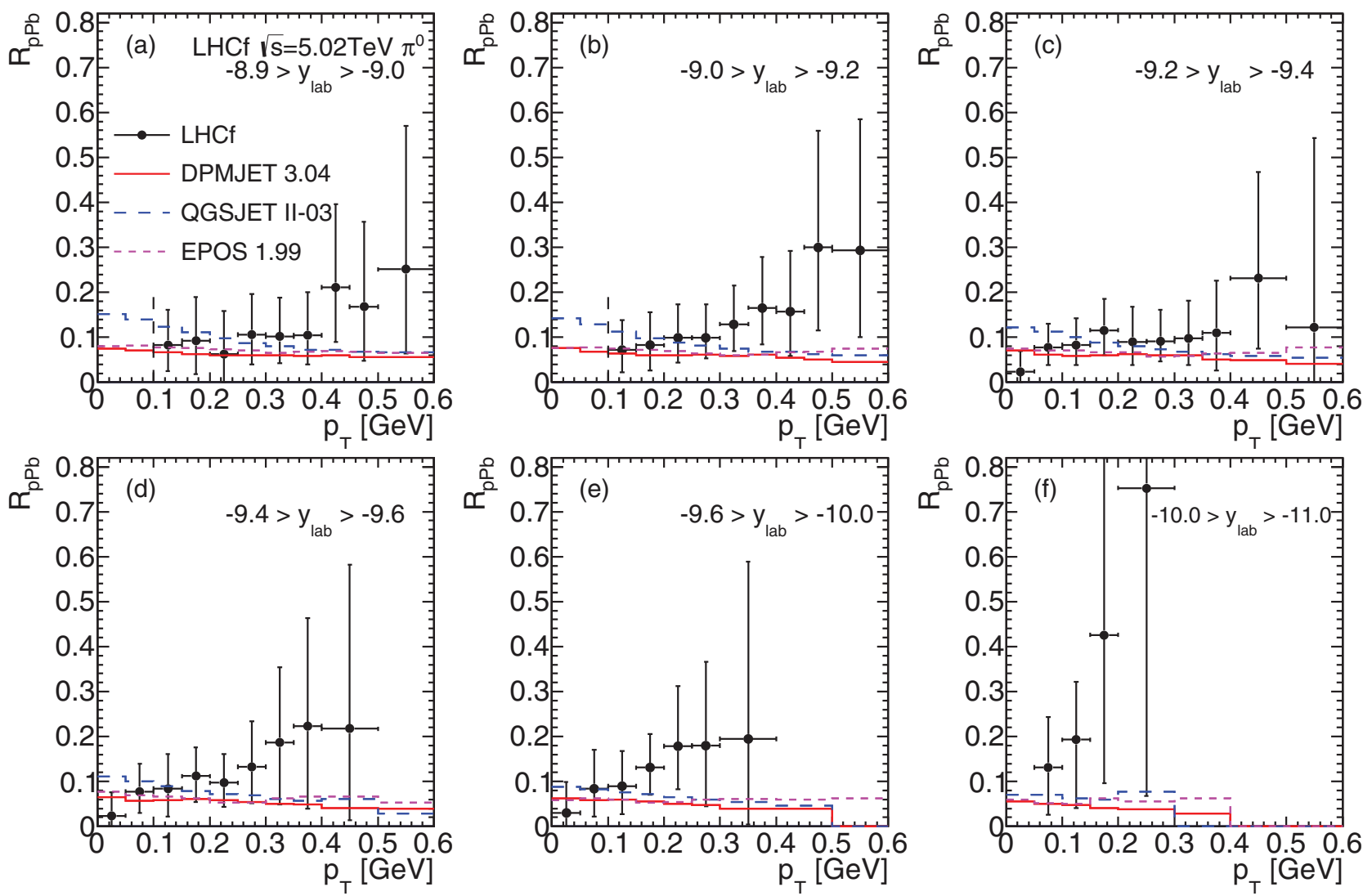

FIG. 6. (Color online) Nuclear modification factor for $\pi^{0}$ 's. Filled circles indicate the factors obtained by the LHCf measurements. Error bars indicate the total uncertainties incorporating both statistical and systematic uncertainties. Other lines are the predictions by hadronic interaction models (see text for details.) 
TABLE IV. Production rate for the $\pi^{0}$ production in the rapidity range $-8.9>y_{\mathrm{lab}}>-9.0$.

\begin{tabular}{lrr}
\hline \hline$p_{\mathrm{T}}$ range $(\mathrm{GeV})$ & Production rate $\left(\mathrm{GeV}^{-2}\right)$ & Syst+Stat uncertainty $\left(\mathrm{GeV}^{-2}\right)$ \\
\hline$[0.10,0.15]$ & $1.17 \times 10^{-1}$ & $-7.83 \times 10^{-2},+8.91 \times 10^{-2}$ \\
{$[0.15,0.20]$} & $1.00 \times 10^{-1}$ & $-7.75 \times 10^{-2},+8.79 \times 10^{-2}$ \\
{$[0.20,0.25]$} & $4.90 \times 10^{-2}$ & $-5.57 \times 10^{-2},+6.43 \times 10^{-2}$ \\
{$[0.25,0.30]$} & $5.37 \times 10^{-2}$ & $-3.18 \times 10^{-2},+3.85 \times 10^{-2}$ \\
{$[0.30,0.35]$} & $3.09 \times 10^{-2}$ & $-1.69 \times 10^{-2},+2.11 \times 10^{-2}$ \\
{$[0.35,0.40]$} & $1.76 \times 10^{-2}$ & $-1.02 \times 10^{-2},+1.24 \times 10^{-2}$ \\
{$[0.40,0.45]$} & $1.82 \times 10^{-2}$ & $-9.42 \times 10^{-3},+1.12 \times 10^{-2}$ \\
{$[0.45,0.50]$} & $6.77 \times 10^{-3}$ & $-4.49 \times 10^{-3},+5.24 \times 10^{-3}$ \\
{$[0.50,0.60]$} & $2.78 \times 10^{-3}$ & $-1.93 \times 10^{-3},+2.20 \times 10^{-3}$ \\
\hline \hline
\end{tabular}

experiment in $p-\mathrm{Pb}$ collisions at $\sqrt{s_{N N}}=5.02 \mathrm{TeV}$ at the LHC in 2013. Transverse momentum spectra of neutral pions measured by the LHCf detectors have been compared with the predictions of several hadronic interaction models. Among the hadronic interaction models tested in this paper, DPMJET 3.04 and EPOS 1.99 show the best overall agreement with the LHCf data in the rapidity range $-8.9>y_{\text {lab }}>-11.0$, while QGSJET II-03 shows softer $p_{\mathrm{T}}$ spectra relative to the the LHCf data and the other two hadronic interaction models. These tendencies are also recognized in the comparison of the average $p_{\mathrm{T}}$ distribution as a function of rapidity $y_{\text {lab }}$.

The nuclear modification factor, $R_{\mathrm{pPb}}$, derived from the LHCf measurements indicates a strong suppression of the $\pi^{0}$ production in the nuclear target relative to those in the nucleon target. All hadronic interaction models present an overall good agreement with the LHCf measurements within the uncertainty.

As a future prospect, additional analyses which are sensitive to exclusive $\pi^{0}$ spectra are needed to reach a better understanding of this strong suppression and its breakdown.

\section{ACKNOWLEDGMENTS}

We thank the CERN staff and the ATLAS Collaboration for their essential contributions to the successful operation of LHCf. This work is partly supported by a Grant-in-Aid for Scientific research by MEXT of Japan, a Grant-in-Aid for a JSPS Postdoctoral Fellowship for Research Abroad, and a Grant-in-Aid for Nagoya University GCOE "QFPU” from MEXT. This work is also supported by Istituto Nazionale di Fisica Nucleare (INFN) in Italy. A part of this work was performed using the computer resources provided by the Institute for the Cosmic-Ray Research (University of Tokyo), CERN, and CNAF (INFN).

\section{APPENDIX A}

The inclusive production rates of $\pi^{0}$ 's measured by LHCf after the subtraction of the UPC component are summarized in Tables IV-IX.

\section{APPENDIX B: DERIVATION OF THE $p_{\mathrm{T}}$ SPECTRA IN $p$ - $p$ COLLISIONS AT $\sqrt{s}=5.02 \mathrm{TeV}$}

To investigate the nuclear effects involved in the nuclear target it is essential to compare the $p_{\mathrm{T}}$ spectra measured in $p$ - $\mathrm{Pb}$ collisions at a given collision energy to the reference $p_{\mathrm{T}}$ spectra in $p$ - $p$ collisions at the same collision energy. In this analysis, since a measurement in $p$ - $p$ collisions at $\sqrt{s}=$ $5.02 \mathrm{TeV}$ is not available, the reference $p_{\mathrm{T}}$ spectra are made by scaling the $p_{\mathrm{T}}$ spectra measured in the $p$ - $p$ collisions at $\sqrt{s}=7$ and $2.76 \mathrm{TeV}$. First the average $p_{\mathrm{T}}$ at $5.02 \mathrm{TeV}$ is estimated by scaling the average $p_{\mathrm{T}}$ obtained at 7 and $2.76 \mathrm{TeV}$. Figure 7 shows the average $p_{\mathrm{T}}$ in $p$ - $p$ collisions at $\sqrt{s}=7 \mathrm{TeV}$ (filled circles) and $2.76 \mathrm{TeV}$ (open circles) as a function of rapidity loss $\Delta y \equiv y_{\text {beam }}-y_{\text {cms }}$, where $y_{\text {beam }}$ is beam rapidity and $y_{\mathrm{cms}}$ is the rapidity of the center-of-mass frame. For the proton beam with $E=3.5$ and $1.38 \mathrm{TeV}, y_{\text {beam }}$ gives 8.917 and 7.987 , respectively. In the following we assume $y_{\mathrm{cms}}$ is positive.

According to the scaling law proposed by several authors [38-40] (Feynman scaling), the average $p_{\mathrm{T}}$ as a function

TABLE V. Production rate for the $\pi^{0}$ production in the rapidity range $-9.0>y_{\text {lab }}>-9.2$.

\begin{tabular}{lrr}
\hline \hline$p_{\mathrm{T}}$ range $(\mathrm{GeV})$ & Production rate $\left(\mathrm{GeV}^{-2}\right)$ & Syst+Stat uncertainty $\left(\mathrm{GeV}^{-2}\right)$ \\
\hline$[0.10,0.15]$ & $9.06 \times 10^{-2}$ & $-6.05 \times 10^{-2},+6.61 \times 10^{-2}$ \\
{$[0.15,0.20]$} & $7.71 \times 10^{-2}$ & $-4.92 \times 10^{-2},+5.54 \times 10^{-2}$ \\
{$[0.20,0.25]$} & $6.28 \times 10^{-2}$ & $-3.23 \times 10^{-2},+3.84 \times 10^{-2}$ \\
{$[0.25,0.30]$} & $3.83 \times 10^{-2}$ & $-1.58 \times 10^{-2},+2.31 \times 10^{-2}$ \\
{$[0.30,0.35]$} & $2.81 \times 10^{-2}$ & $-1.13 \times 10^{-2},+1.36 \times 10^{-2}$ \\
{$[0.35,0.40]$} & $1.82 \times 10^{-2}$ & $-7.76 \times 10^{-3},+8.27 \times 10^{-3}$ \\
{$[0.40,0.45]$} & $7.98 \times 10^{-3}$ & $-4.49 \times 10^{-3},+4.33 \times 10^{-3}$ \\
{$[0.45,0.50]$} & $6.37 \times 10^{-3}$ & $-3.43 \times 10^{-3},+3.05 \times 10^{-3}$ \\
{$[0.50,0.60]$} & $1.41 \times 10^{-3}$ & $-8.01 \times 10^{-4},+6.56 \times 10^{-4}$ \\
\hline \hline
\end{tabular}


TABLE VI. Production rate for the $\pi^{0}$ production in the rapidity range $-9.2>y_{\text {lab }}>-9.4$.

\begin{tabular}{lrr}
\hline \hline$p_{\mathrm{T}}$ range $(\mathrm{GeV})$ & Production rate $\left(\mathrm{GeV}^{-2}\right)$ & Syst+Stat uncertainty $(\mathrm{GeV}-2)$ \\
\hline$[0.00,0.05]$ & $3.56 \times 10^{-2}$ & $-5.14 \times 10^{-2},+5.46 \times 10^{-2}$ \\
{$[0.05,0.10]$} & $1.02 \times 10^{-1}$ & $-4.63 \times 10^{-2},+4.99 \times 10^{-2}$ \\
{$[0.10,0.15]$} & $8.53 \times 10^{-2}$ & $-4.15 \times 10^{-2},+4.49 \times 10^{-2}$ \\
{$[0.15,0.20]$} & $8.25 \times 10^{-2}$ & $-3.45 \times 10^{-2},+3.67 \times 10^{-2}$ \\
{$[0.20,0.25]$} & $3.96 \times 10^{-2}$ & $-2.11 \times 10^{-2},+2.83 \times 10^{-2}$ \\
{$[0.25,0.30]$} & $2.19 \times 10^{-2}$ & $-9.62 \times 10^{-3},+1.26 \times 10^{-2}$ \\
{$[0.30,0.35]$} & $1.14 \times 10^{-2}$ & $-6.25 \times 10^{-3},+6.81 \times 10^{-3}$ \\
{$[0.35,0.40]$} & $5.53 \times 10^{-3}$ & $-3.93 \times 10^{-3},+3.75 \times 10^{-3}$ \\
{$[0.40,0.50]$} & $2.67 \times 10^{-3}$ & $-1.57 \times 10^{-3},+1.33 \times 10^{-3}$ \\
{$[0.50,0.60]$} & $1.31 \times 10^{-4}$ & $-2.45 \times 10^{-4},+2.36 \times 10^{-4}$ \\
\hline \hline
\end{tabular}

TABLE VII. Production rate for the $\pi^{0}$ production in the rapidity range $-9.4>y_{\text {lab }}>-9.6$.

\begin{tabular}{lcr}
\hline \hline$p_{\mathrm{T}}$ range $(\mathrm{GeV})$ & Production rate $\left(\mathrm{GeV}^{-2}\right)$ & Syst+Stat uncertainty $(\mathrm{GeV}-2)$ \\
\hline$[0.00,0.05]$ & $2.67 \times 10^{-2}$ & $-4.42 \times 10^{-2},+4.64 \times 10^{-2}$ \\
{$[0.05,0.10]$} & $7.42 \times 10^{-2}$ & $-4.11 \times 10^{-2},+4.31 \times 10^{-2}$ \\
{$[0.10,0.15]$} & $5.87 \times 10^{-2}$ & $-4.08 \times 10^{-2},+4.19 \times 10^{-2}$ \\
{$[0.15,0.20]$} & $4.93 \times 10^{-2}$ & $-2.26 \times 10^{-2},+2.13 \times 10^{-2}$ \\
{$[0.20,0.25]$} & $2.32 \times 10^{-2}$ & $-1.17 \times 10^{-2},+1.16 \times 10^{-2}$ \\
{$[0.25,0.30]$} & $1.47 \times 10^{-2}$ & $-8.10 \times 10^{-3},+7.54 \times 10^{-3}$ \\
{$[0.30,0.35]$} & $8.37 \times 10^{-3}$ & $-5.73 \times 10^{-3},+4.31 \times 10^{-3}$ \\
{$[0.35,0.40]$} & $3.47 \times 10^{-3}$ & $-2.68 \times 10^{-3},+1.77 \times 10^{-3}$ \\
{$[0.40,0.50]$} & $5.32 \times 10^{-4}$ & $-4.86 \times 10^{-4},+3.39 \times 10^{-4}$ \\
\hline \hline
\end{tabular}

TABLE VIII. Production rate for the $\pi^{0}$ production in the rapidity range $-9.6>y_{\text {lab }}>-10.0$.

\begin{tabular}{lrr}
\hline \hline$p_{\mathrm{T}}$ range $(\mathrm{GeV})$ & Production rate $\left(\mathrm{GeV}^{-2}\right)$ & Syst+Stat uncertainty $(\mathrm{GeV}-2)$ \\
\hline$[0.00,0.05]$ & $1.72 \times 10^{-2}$ & $-2.93 \times 10^{-2},+3.07 \times 10^{-2}$ \\
{$[0.05,0.10]$} & $3.93 \times 10^{-2}$ & $-2.69 \times 10^{-2},+2.83 \times 10^{-2}$ \\
{$[0.10,0.15]$} & $2.67 \times 10^{-2}$ & $-1.72 \times 10^{-2},+1.81 \times 10^{-2}$ \\
{$[0.15,0.20]$} & $2.00 \times 10^{-2}$ & $-8.16 \times 10^{-3},+8.44 \times 10^{-3}$ \\
{$[0.20,0.25]$} & $1.13 \times 10^{-2}$ & $-5.12 \times 10^{-3},+5.06 \times 10^{-3}$ \\
{$[0.25,0.30]$} & $3.83 \times 10^{-3}$ & $-2.61 \times 10^{-3},+1.85 \times 10^{-3}$ \\
{$[0.30,0.40]$} & $5.56 \times 10^{-4}$ & $-5.35 \times 10^{-4},+3.86 \times 10^{-4}$ \\
\hline \hline
\end{tabular}

TABLE IX. Production rate for the $\pi^{0}$ production in the rapidity range $-10.0>y_{\text {lab }}>-11.0$.

\begin{tabular}{lcr}
\hline \hline$p_{\mathrm{T}}$ range $(\mathrm{GeV})$ & Production rate $\left(\mathrm{GeV}^{-2}\right)$ & Syst+Stat uncertainty $(\mathrm{GeV}-2)$ \\
\hline$[0.00,0.05]$ & $-6.79 \times 10^{-3}$ & $-7.16 \times 10^{-3},+7.43 \times 10^{-3}$ \\
{$[0.05,0.10]$} & $6.12 \times 10^{-3}$ & $-4.74 \times 10^{-3},+3.51 \times 10^{-3}$ \\
{$[0.10,0.15]$} & $3.51 \times 10^{-3}$ & $-2.66 \times 10^{-3},+1.54 \times 10^{-3}$ \\
{$[0.15,0.20]$} & $2.01 \times 10^{-3}$ & $-1.39 \times 10^{-3},+8.46 \times 10^{-4}$ \\
{$[0.20,0.30]$} & $2.36 \times 10^{-4}$ & $-2.00 \times 10^{-4},+1.08 \times 10^{-4}$ \\
\hline \hline
\end{tabular}




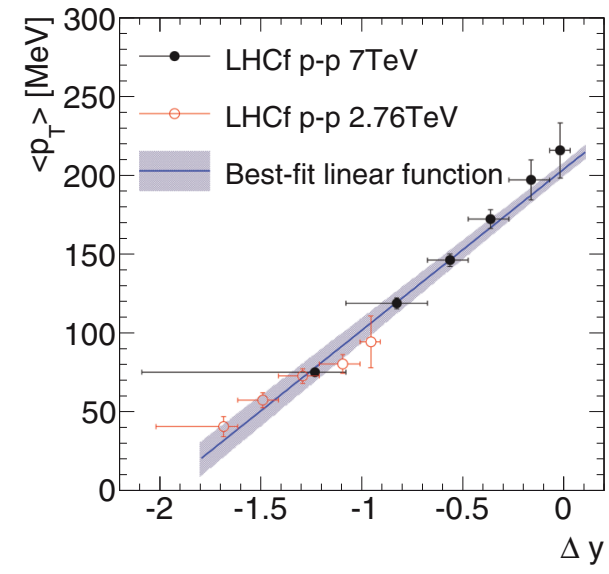

FIG. 7. (Color online) Average $p_{\mathrm{T}}$ as a function of rapidity loss $\Delta y$. Filled circles indicate the LHCf results in $p$ - $p$ collisions at $\sqrt{s}=$ $7 \mathrm{TeV}$ taken from Ref. [29]. Open circles indicate the LHCf results in $p$ - $p$ collisions at $\sqrt{s}=2.76 \mathrm{TeV}$. The best-fit linear function to the LHCf data is shown by the solid line.

of $\Delta y$ should be independent of the center-of-mass energy in the projectile fragmentation region. Thus the average $p_{\mathrm{T}}$ can be directly compared among different collision energies. The values of the average $p_{\mathrm{T}}$ at $7 \mathrm{TeV}$ are taken from measurements by LHCf [29] in which the associated $\Delta y$ points are modified to take into account event population for each rapidity bin. These weighted bin centers are estimated using the MC simulation by EPOS 1.99 . The values of the average $p_{\mathrm{T}}$ at $2.76 \mathrm{TeV}$ are obtained by a similar analysis of the data that was taken in $p$ - $p$ collisions at $\sqrt{s}=2.76 \mathrm{TeV}$ on February 13, 2013. These data were taken with essentially the same data acquisition configuration as at $5.02 \mathrm{TeV}$.

Although the two measurements in Fig. 7 have limited overlap on the $\Delta y$ range owing to the smaller collision energy at $2.76 \mathrm{TeV}$, the $\left\langle p_{\mathrm{T}}\right\rangle$ spectra at 7 and $2.76 \mathrm{TeV}$ follow mostly a common line. A linear function fit is then made to these measurements. The solid line and shaded area in Fig. 7 show the best-fit linear function and the one-standarddeviation uncertainty obtained by a chi-square fit to the data points,

$$
\left\langle p_{\mathrm{T}}\right\rangle_{\text {best-fit }}(\Delta y)=216.3+116.0 \Delta y .
$$

The minimum chi-square value is 11.1 with a number of degrees of freedom equal to 9. With the fitted result in Eq. (B1) and $y_{\text {beam }}=8.585$ for the proton beam with $E=2.51 \mathrm{TeV}$, the average $p_{\mathrm{T}}$ at a given rapidity $y_{\mathrm{cms}}$ at $5.02 \mathrm{TeV}$ can be evaluated as

$$
\left.\left\langle p_{\mathrm{T}}\right\rangle\left(y_{\mathrm{cms}}\right)\right|_{5.02 \mathrm{TeV}}=216.3+116.0\left(8.585-y_{\mathrm{cms}}\right),
$$

where we assume the proton beam travels to the positive rapidity direction. Note that the rapidity range of the reference $p_{\mathrm{T}}$ spectra at $5.02 \mathrm{TeV}$ is enclosed by the data points taken at 7 and $2.76 \mathrm{TeV}$.

The absolute normalization scaling among three collisions is then estimated for $2.76,5.02$, and $7 \mathrm{TeV}$ energies. Since the systematic uncertainty of the LHCf measurements on the luminosity is $\pm 20 \%$ and $\pm 6.1 \%$ at 2.76 and $7 \mathrm{TeV}$ respectively, the predictions by MC simulations are used instead for the estimation. According to DPMJET 3.04, QGSJET II-03, and EPOS 1.99 , the relative normalization at $5.02 \mathrm{TeV}$ to that at 7 or $2.76 \mathrm{TeV}$, defined as

$$
\begin{aligned}
R_{\mathrm{norm}} \equiv & \left.\int d p^{3} \frac{1}{\sigma_{\text {inel }}} E \frac{d^{3} \sigma}{d p^{3}}\right|_{\sqrt{s}=5.02 \mathrm{TeV}} / \\
& \left.\int d p^{3} \frac{1}{\sigma_{\text {inel }}} E \frac{d^{3} \sigma}{d p^{3}}\right|_{\sqrt{s}=7 \text { or } 2.76 \mathrm{TeV}}
\end{aligned}
$$

is mostly unity in the rapidity and $p_{\mathrm{T}}$ ranges covered by LHCf. Therefore we apply the measured absolute normalization at $7 \mathrm{TeV}$ to the reference $p_{\mathrm{T}}$ spectra at $5.02 \mathrm{TeV}$ without scaling. The uncertainty on the normalization is taken from the luminosity error $\pm 6.1 \%$.

Accordingly the average $p_{\mathrm{T}}$ and normalization of $p_{\mathrm{T}}$ spectra at $5.02 \mathrm{TeV}$ can be scaled from 7 and $2.76 \mathrm{TeV}$. With these two values, the $p_{\mathrm{T}}$ spectra in $p$ - $p$ collisions at $5.02 \mathrm{TeV}$ can be effectively derived. In the analysis of this paper, the expected $p_{\mathrm{T}}$ spectra are presumed to follow a Gaussian distribution with the width of the distribution $\sigma_{\text {Gauss }}$ equal to $2\left\langle p_{\mathrm{T}}\right\rangle / \sqrt{\pi}$. The expected $p_{\mathrm{T}}$ spectra in $p-p$ collisions take into account the rapidity shift -0.465 explained in Sec. III for consistency with the asymmetric beam energies in $p$ - $\mathrm{Pb}$ collisions.
[1] I. Abt et al. (H1 Collaboration), Nucl. Phys. B 407, 515 (1993).

[2] M. Derrick et al. (ZEUS Collaboration), Phys. Lett. B 316, 412 (1993).

[3] I. Arsene et al. (BRAHMS Collaboration), Phys. Rev. Lett 93, 242303 (2004).

[4] J. Adams et al. (STAR Collaboration), Phys. Rev. Lett 97, 152302 (2006).

[5] B. Abelev et al. (ALICE Collaboration), Phys. Rev. Lett. 110, 082302 (2013).

[6] R. Aaij et al. (LHCb Collaboration), J. High Energy Phys. 02 (2014) 072.
[7] A. Dumitru, L. Gerland, and M. Strikman, Phys. Rev. Lett. 90, 092301 (2003).

[8] J. Jalilian-Marian and A. H. Rezaeian, Phys. Rev D 85, 014017 (2012).

[9] J. L. Albacete, A. Dumitrub, H. Fujii, and Y. Nara, Nucl. Phys. A 897, 1 (2013).

[10] A. Letessier-Selvon and T. Stanev, Rev. Mod. Phys. 83, 907 (2011).

[11] LHCf Technical Design Report No. CERN-LHCC-2006-004 (unpublished).

[12] O. Adriani et al. (LHCf Collaboration), JINST 3, S08006 (2008). 
[13] O. Adriani et al. (LHCf Collaboration), JINST 5, P01012 (2010).

[14] W. C. Turner, E. H. Hoyer, and N. V. Mokhov, in Proceedings of EPAC98, Stockholm, 1998 (unpublished), p. 368, http:// accelconf.web.cern.ch/AccelConf/e98/contents.html; LBNL Report No. LBNL-41964, 1998 (unpublished).

[15] T. Mase et al. (LHCf Collaboration), Nucl. Instrum. Methods A 671, 129 (2012).

[16] O. Adriani et al. (LHCf Collaboration), Int. J. Mod. Phys. A 28, 1330036 (2013).

[17] LHC Performance and Statistics, https://lhc-statistics.web. cern.ch/LHC-Statistics/.

[18] O. Adriani et al. (LHCf Collaboration), Phys. Lett. B 703, 128 (2011).

[19] J. M. Jowett et al., in Proceedings of IPAC2013, edited by Z. Dai, C. Petit-Jean-Genaz, V. RW Schaa, and C. Zhang (JACoW, Shanghai, 2013).

[20] F. W. Bopp, J. Ranft, R. Engel, and S. Roesler, Phys. Rev. C 77, 014904 (2008).

[21] S. Ostapchenko, Nucl. Phys. Proc. Suppl. 151, 143 (2006).

[22] K. Werner, F.-M. Liu, and T. Pierog, Phys. Rev. C 74, 044902 (2006).

[23] J. W. Cronin, H. J. Frisch, M. J. Shochet, J. P. Boymond, P. A. Piroué, and R. L. Sumner, Phys. Rev. D 11, 3105 (1975).

[24] C. A. Bertulani, S. R. Klein, and J. Nystrand, Annu. Rev. Nucl. Part. Sci. 55, 271 (2005).

[25] C. F. v. Weizsäcker, Z. Phys. 88, 612 (1934); E. J. Williams, Phys. Rev. 45, 729 (1934).
[26] A. Mücke, R. Engel, J. P. Rachen, R. J. Protheroe, and T. Stanev, Comput. Phys. Commun. 124, 290 (2000).

[27] K. Kasahara, in Proceedings of the 24th International Cosmic Ray Conference, Rome, 1995 (unpublished). EPICS web page, http://cosmos.n.kanagawa-u.ac.jp/.

[28] K. Kawade et al. (LHCf Collaboration), JINST 9, P03016 (2014).

[29] O. Adriani et al. (LHCf Collaboration), Phys. Rev. D. 86, 092001 (2012)

[30] G. D'Agostini, Nucl. Instrum. Methods A 362, 487 (1995)

[31] G. Vismara, CERN Report No. CERN-SL-2000-056 BI, 2000 (unpublished).

[32] R. J. Glauber and G. Matthiae, Nucl. Phys. B 21, 135 (1970).

[33] D. d'Enterria, arXiv:nucl-ex/0302016. Updated information is available at http://dde.web.cern.ch/dde/glauber_lhc.htm.

[34] O. Adriani et al. (LHCf Collaboration) (unpublished).

[35] The CMS Collaboration, CMS Report No. CMS-PAS-FSQ-13006 (unpublished).

[36] B. Abelev et al. (ALICE Collaboration), arXiv:1405.1849, CERN Report No. CERN-PH-EP-2014-031.

[37] B. Abelev et al. (ALICE Collaboration), Phys. Rev. Lett. 110, 032301 (2013).

[38] D. Amati, A. Stanghellini, and S. Fubini, Nuovo Cimento 26, 896 (1962).

[39] J. Benecke, T. T. Chou, C. N. Yang, and E. Yen, Phys. Rev. 188, 2159 (1969).

[40] R. P. Feynman, Phys. Rev. Lett. 23, 1415 (1969).

O. Adriani, ${ }^{1,2}$ E. Berti, ${ }^{1,2}$ L. Bonechi, ${ }^{1}$ M. Bongi, ${ }^{1,2}$ G. Castellini, ${ }^{1,3}$ R. D’Alessandro, ${ }^{1,2}$ M. Del Prete, ${ }^{1,2}$ M. Haguenauer, ${ }^{4}$ Y. Itow, ${ }^{5,6}$ K. Kasahara, ${ }^{7}$ K. Kawade, ${ }^{5}$ Y. Makino, ${ }^{5}$ K. Masuda, ${ }^{5}$ E. Matsubayashi, ${ }^{5}$ H. Menjo, ${ }^{8}$ G. Mitsuka, ${ }^{2,5}$ Y. Muraki, ${ }^{5}$ P. Papini, ${ }^{1}$ A.-L. Perrot, ${ }^{9}$ D. Pfeiffer, ${ }^{9}$ S. Ricciarini, ${ }^{1,3}$ T. Sako, ${ }^{5,6}$ N. Sakurai, ${ }^{6}$ T. Suzuki, ${ }^{7}$ T. Tamura, ${ }^{10}$ A. Tiberio, ${ }^{1,2}$ S. Torii, ${ }^{7}$ A. Tricomi, ${ }^{11,12}$ and W. C. Turner ${ }^{13}$

(LHCf Collaboration)

\author{
${ }^{1}$ INFN Section of Florence, Florence, Italy \\ ${ }^{2}$ University of Florence, Florence, Italy \\ ${ }^{3}$ IFAC-CNR, Florence, Italy \\ ${ }^{4}$ Ecole-Polytechnique, Palaiseau, France
}

${ }^{5}$ Solar-Terrestrial Environment Laboratory, Nagoya University, Nagoya, Japan

${ }^{6}$ Kobayashi-Maskawa Institute for the Origin of Particles and the Universe, Nagoya University, Nagoya, Japan

${ }^{7}$ RISE, Waseda University, Shinjuku, Tokyo, Japan

${ }^{8}$ Graduate School of Science, Nagoya University, Nagoya, Japan

${ }^{9}$ CERN, Geneva, Switzerland

${ }^{10}$ Kanagawa University, Kanagawa, Japan

${ }^{11}$ INFN Section of Catania, Catania, Italy

${ }^{12}$ University of Catania, Catania, Italy

${ }^{13}$ LBNL, Berkeley, California, USA 